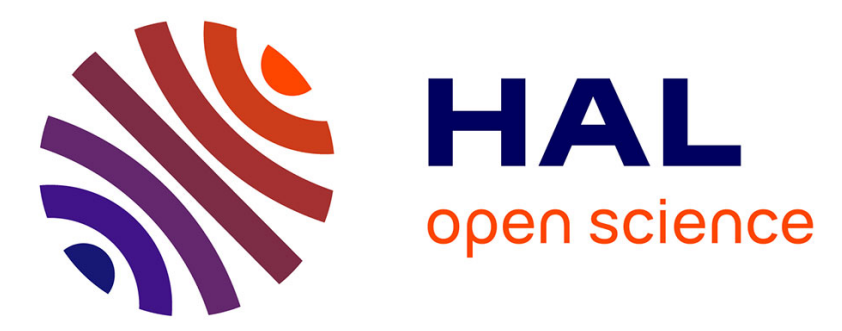

\title{
Reactivity of benzofuran and benzothiophene in palladium-catalysed direct C2,C3-diarylations
}

Karima Si Larbi, Safia Djebbar, Jean-François Soulé, Henri Doucet

\section{To cite this version:}

Karima Si Larbi, Safia Djebbar, Jean-François Soulé, Henri Doucet. Reactivity of benzofuran and benzothiophene in palladium-catalysed direct C2,C3-diarylations. Journal of Organometallic Chemistry, 2017, 843, pp.32-39. 10.1016/j.jorganchem.2017.05.029 . hal-01559209

HAL Id: hal-01559209

https://hal-univ-rennes1.archives-ouvertes.fr/hal-01559209

Submitted on 13 Oct 2017

HAL is a multi-disciplinary open access archive for the deposit and dissemination of scientific research documents, whether they are published or not. The documents may come from teaching and research institutions in France or abroad, or from public or private research centers.
L'archive ouverte pluridisciplinaire HAL, est destinée au dépôt et à la diffusion de documents scientifiques de niveau recherche, publiés ou non, émanant des établissements d'enseignement et de recherche français ou étrangers, des laboratoires publics ou privés. 


\section{Graphical Abstract}

Reactivity of benzofuran and Leave this area blank for abstract info.

benzothiophene in palladium-catalysed

direct C2,C3-diarylations

Karima Si Larbi, Safia Djebbar, Jean-François Soulé, Henri Doucet

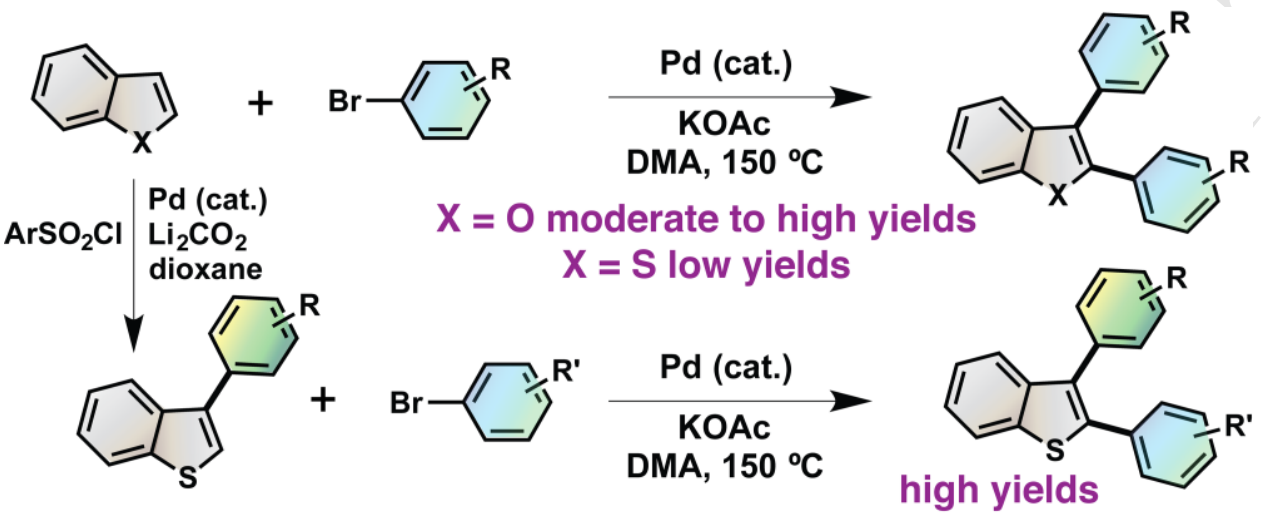




\title{
Reactivity of benzofuran and benzothiophene in palladium-catalysed direct C2,C3- diarylations
}

\author{
Karima Si Larbi, ${ }^{[a, b]}$ Safia Djebbar, ${ }^{[a, b]}$ Jean-François Soulé, ${ }^{[a]}$ Henri Doucet ${ }^{*[a]}$ \\ a Institut des Sciences Chimiques de Rennes, UMR 6226, CNRS-Université de Rennes 1", Organométalliques, matériaux et Catalyse", Campus de Beaulieu, \\ 35042 Rennes, France, E-mail: jean-francois.soule@univ-rennes1.fr; henri.doucet@univ-rennes1.fr \\ ${ }^{b}$ Laboratoire d'hydrométallurgie et chimie inorganique moléculaire, Faculté de Chimie, U.S.T.H.B. Bab-Ezzouar, Algeria.
}

\section{ARTICLE INFO}

\section{ABSTRACT}

\section{Article history:}

Received

Received in revised form

Accepted

Available online

Keywords:

Palladium

Catalysis

$\mathrm{C}-\mathrm{H}$ bond functionalization

Benzofuran

Benzothiophene

\section{Introduction}

The arylation of heteroaromatics such as benzofuran and benzothiophene is an important field for research in organic chemistry due to the biological properties of some of their derivatives. For example, Saprisartan is an AT1 receptor antagonist, Furaprofen is a non-steroidal anti-inflammatory drug and Raloxifene is used in the prevention of osteoporosis (Fig. 1).

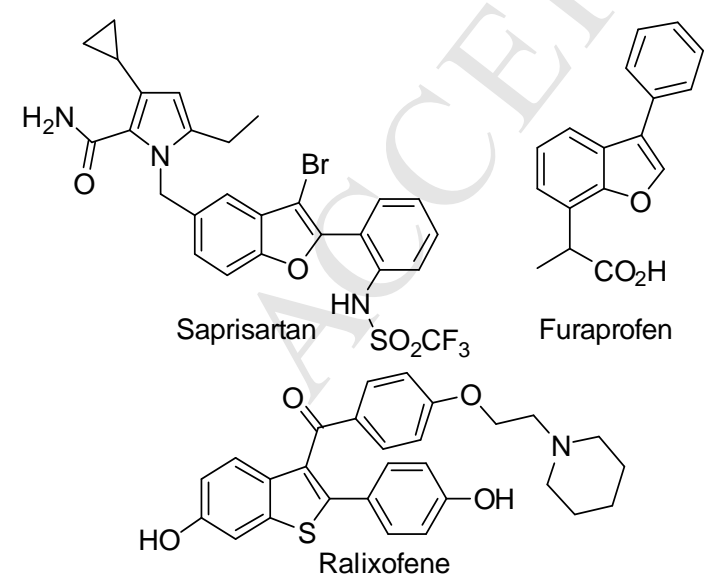

Figure 1. Examples of bioactive aryl-substituted benzofurans and benzothiophenes

In 1990, Ohta and co-workers reported that the C2-arylation of several heteroaromatics, including benzofuran and benzothiophene, with aryl bromides, via a $\mathrm{C}-\mathrm{H}$ bond activation, proceed in moderate to good yields using $\mathrm{Pd}\left(\mathrm{PPh}_{3}\right)_{4}$ as the catalyst. ${ }^{1} \quad$ Since these seminal results, the palladium-catalysed so-called direct arylation of heteroaryl derivatives proved to be an extremely reliable method for the synthesis of a wide variety of arylated heterocycles. ${ }^{2}$

For the Pd-catalysed direct arylation of most 5-membered ring heterocycles, including benzofuran ${ }^{3}$ and benzothiophene, ${ }^{4}$ the reaction often proceeds via a concerted metallation deprotonation (CMD) mechanism. ${ }^{5}$ According to Gorelsky calculations, in the CMD process, the carbon 2 of benzofuran should be slightly more reactive than carbon 3 (energies: 26.3 vs 27.5); whereas for benzothiophene $\mathrm{C} 2$-position is much more reactive than $\mathrm{C} 3$ position (energies: 26.5 vs 29.2) (Fig. 2). ${ }^{6}$ Therefore, the one pot C2,C3-diarylation of these two heteroaromatic compounds should be possible, but benzothiophene is expected to be less reactive than benzofuran.

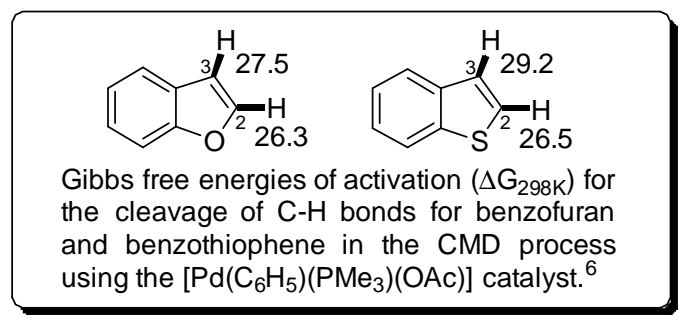

Figure 2. Benzofuran and benzothiophene Gibbs free energies of activation for CMD process.

So far, relatively little efforts have been expended toward developing such Pd-catalysed direct arylation reactions for the one pot synthesis of 2,3-diarylated benzofurans or 
benzothiophenes. $^{7-9}$ These compounds are generally prepared via Suzuki reactions. ${ }^{10,11}$ The first example of palladiumcatalysed direct diarylation at carbons $\mathrm{C} 2$ and $\mathrm{C} 3$ of benzofuran was reported by Daugulis and Chiong in 2007. They prepared 2,3-diphenylbenzofuran in $68 \%$ yield, using chlorobenzene as the coupling partner and $5 \mathrm{~mol} \% \mathrm{Pd}(\mathrm{OAc})_{2}$ associated to $10 \mathrm{~mol} \%$ $\mathrm{P} n \mathrm{Bu}(\mathrm{Ad})_{2}$ as the catalyst system (Scheme 1, a). ${ }^{7 \mathrm{a}}$ Similarly, Shibahara, Murai et al. reported in 2010 an example of 2,3diarylation of benzofuran with 4-(trifluoromethyl)iodobenzene in $51 \%$ yield using $5 \mathrm{~mol} \% \mathrm{Pd}(\mathrm{phen})_{2}\left(\mathrm{PF}_{6}\right)_{2}$ catalyst (Scheme 1, b). ${ }^{7 \mathrm{~b}}$ The last example of diarylation of benzofuran was reported in 2015. Using 3-bromopyridine as aryl source and a bis(alkoxo)palladium complex as catalyst, the 2,3diarylbenzofuran was obtained in $44 \%$ yield together with $47 \%$ of the mono-C2-arylated benzofuran (Scheme 1, c). ${ }^{7 \mathrm{c}}$ A few examples of palladium-catalysed sequential diarylations of benzofuran have also been described. ${ }^{8}$ Concerning benzothiophene, very few examples of C2,C3-diarylations have been reported. Schnürch et al. reported, in the course of their studies on benzothiophene C2-arylation reaction, that in some cases trace amount of diarylations were observed. ${ }^{8 \mathrm{~b}}$ So far, C2,C3-diarylated benzothiophenes have been prepared via successive Pd-catalysed direct arylations. Our laboratory reported in 2013 that from a 2-arylbenzothiophene and 4bromobenzonitrile using $\mathrm{PdCl}\left(\mathrm{C}_{3} \mathrm{H}_{5}\right)(\mathrm{dppb})$ catalyst, the 2,3-

a)
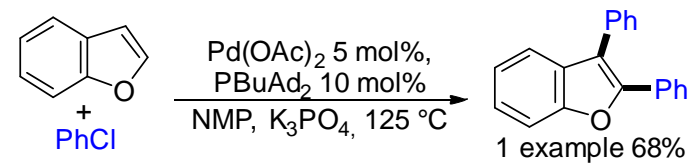

b)<smiles>c1ccc2occc2c1</smiles>
$\frac{\mathrm{Pd}(\mathrm{phen})_{2}\left(\mathrm{PF}_{6}\right)_{2} 5 \mathrm{~mol} \%}{\mathrm{Cs}_{2} \mathrm{CO}_{3}, \mathrm{DMA}, 150{ }^{\circ} \mathrm{C}}$ Arl Ar: $4-\mathrm{CF}_{3} \mathrm{C}_{6} \mathrm{H}_{4}$

c)

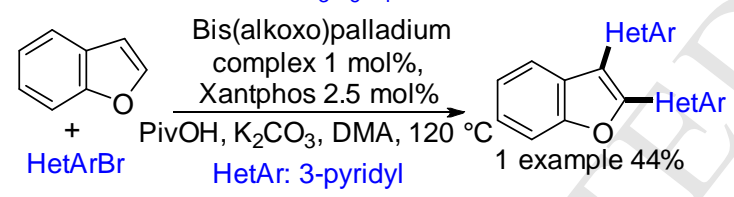

d)<smiles></smiles><smiles>N#Cc1ccc(Br)cc1</smiles>
( 2 equiv.

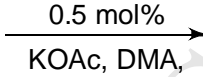

$150^{\circ} \mathrm{C}, 20 \mathrm{~h} \quad 1$ example $47 \%$

e)

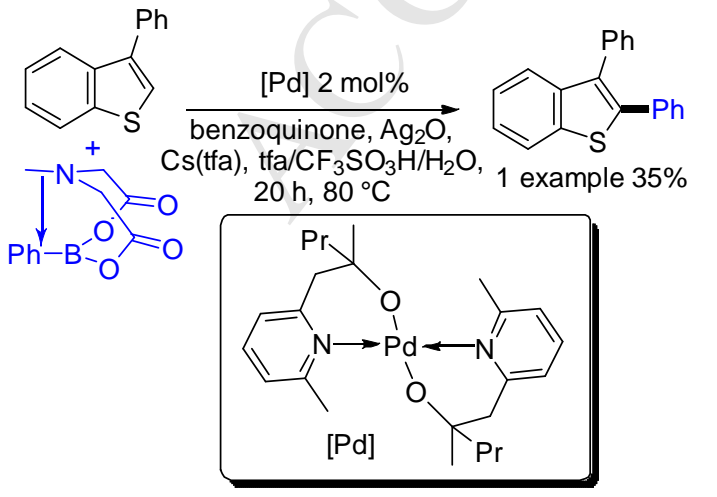

Scheme 1. Reported Pd-catalysed direct diarylations of benzofuran and arylations of arylbenzothiophenes diarylated benzothiophene could be obtained in $47 \%$ yield (Scheme 1, d). ${ }^{9 \mathrm{a}}$ A procedure, using so-called MIDA boronate instead of aryl bromides as coupling partners for C2-arylation of 3-phenylbenzothiophene also led to a C2,C3-diarylated benzothiophene in moderate yield (Scheme 1, e). ${ }^{9 \mathrm{c}}$

Therefore, the reactivity of both benzofuran and benzothiophene in Pd-catalysed direct 2,3-diarylation needed to be investigated. Herein, we describe the reactivity of benzofuran and benzothiophene for access to their C2,C3-diarylated derivatives via one pot reaction or successive arylations. The influence of the (hetero)aryl bromide substituents is also reported.

\section{Results and discussion}

Based on our previous results on palladium-catalysed direct arylation of heteroarenes, ${ }^{2 \mathrm{~m}, 9 \mathrm{a}}$ we first examined the reactivity of benzofuran in the presence of an excess of 4-bromobenzonitrile (Scheme 2). The reaction of 3 equiv. of this aryl bromide with 1 equiv. of benzofuran in the presence of $2 \mathrm{~mol} \%$ $\mathrm{PdCl}_{2}\left(\mathrm{C}_{3} \mathrm{H}_{5}\right)(\mathrm{dppb})$ catalyst and 3 equiv. of KOAc as the base at $150{ }^{\circ} \mathrm{C}$ during $16 \mathrm{~h}$ gave the desired products 1 in $62 \%$. The formation of mono-arylation products in low yield was also detected by GC/MS analysis of the crude mixture (Scheme 2).

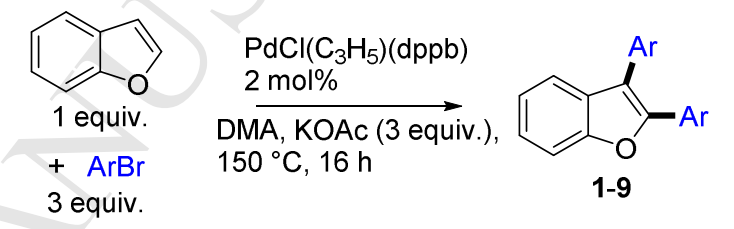

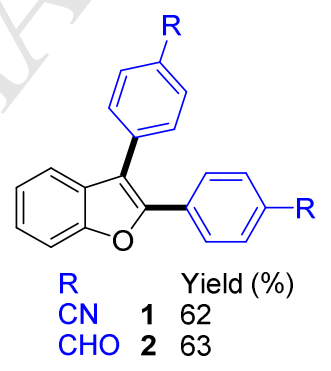

$\mathrm{F}_{3} \mathrm{C}$<smiles>FC(F)(F)c1cc(-c2oc3ccccc3c2-c2cc(C(F)(F)F)cc(C(F)(F)F)c2)cc(C(F)(F)F)c1</smiles><smiles>N#Cc1cccc(-c2oc3ccccc3c2-c2cccc(C#N)c2)c1</smiles>

$364 \%$<smiles>[R]c1ccccc1-c1oc2ccccc2c1-c1ccccc1[R]</smiles>

R $\quad$ Yield (\%)

CN 560

$$
\mathrm{CHO} \quad 6 \quad 57
$$<smiles>c1cncc(-c2oc3ccccc3c2-c2cccnc2)c1</smiles><smiles>FC(F)(F)c1cccc(-c2oc3ccccc3c2-c2cccc(C(F)(F)F)n2)n1</smiles>

$768 \%$<smiles>c1ccc2c(-c3cncnc3)c(-c3cncnc3)oc2c1</smiles>

$973 \%$
Scheme 2. Pd-catalysed direct diarylations of benzofuran

Then, the influence of the substituents on aryl bromide for diarylation of benzofuran, under these reaction conditions, was examined (Scheme 2). For this study, we initially employed para- and meta-substituted aryl bromides. Both 4- 
bromobenzaldehyde and 3-bromobenzonitrile gave the target diarylated benzofurans $\mathbf{2}$ and $\mathbf{3}$ in good yields. Trifluoromethyl substituents at both $\mathrm{C} 3$ and $\mathrm{C} 5$ positions of the aryl bromide gave the C2,C3-diarylated benzofuran 4 in $83 \%$ yield. Nitrile or formyl ortho-substituents on the aryl bromide were also tolerated as the desired products $\mathbf{5}$ and $\mathbf{6}$ were obtained in $60 \%$ and $57 \%$ yields, respectively. Pyridine is probably the most common heterocyclic motif found in pharmaceutically active compounds. It is also one of the most commonly employed motif in ligands used in organometallic chemistry. Therefore, the discovery of preparative methods of derivatives containing pyridines remain an essential research topic in organic synthesis. From benzofuran and 3 equiv. of 3-bromopyridine or 2-bromo-6(trifluoromethyl)pyridine, the diarylated benzofurans $\mathbf{7}$ and $\mathbf{8}$ were obtained in $68 \%$ and $61 \%$ yields, respectively. 5Bromopyrimidine was also tolerated as aryl source, with the formation of 9 in $73 \%$ yield.

The Pd-catalysed one pot direct C2,C3-diarylation of benzothiophene is more challenging as the $\mathrm{C}-\mathrm{H}$ bond cleavage at $\mathrm{C} 3$-position via concerted metallation deprotonation requires an higher Gibbs free energy of activation (see Fig. 2). As expected, in most cases, sluggish reactions were observed with the formation of large amounts of the mono-C2-arylated benzothiophenes (12-41\%) as side-products (Scheme 3). The best yield in C2,C3-diarylated benzothiophene was obtained for the coupling of 3-bromonitrobenzene with the formation of $\mathbf{1 1}$ in $60 \%$ yield; whereas, the reaction of bromobenzaldehydes or bromopyridines afforded 10 and $\mathbf{1 2 - 1 4}$ in $31-42 \%$ yields.

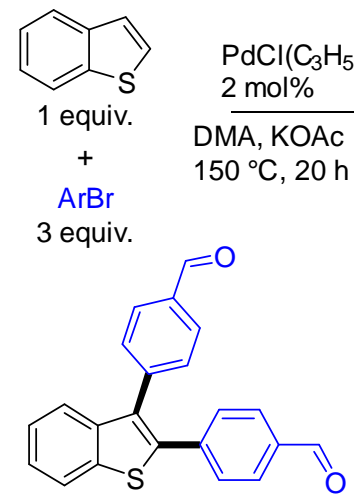

$1042 \%\left(38 \% \%^{*}\right)$
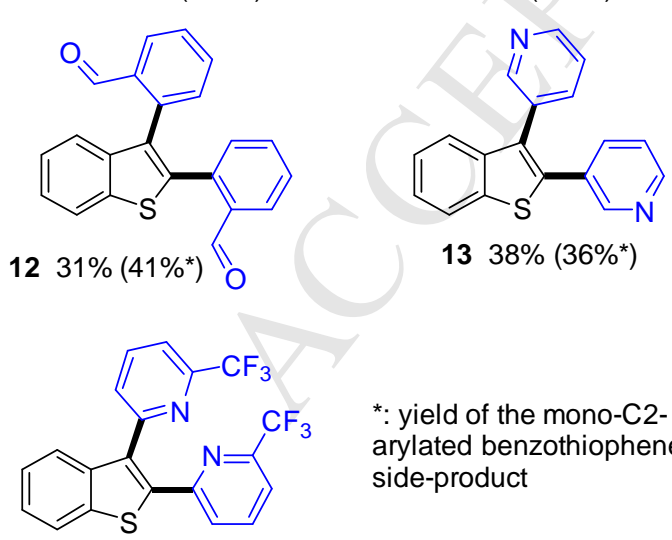

*: yield of the mono-C2-

arylated benzothiophene

side-product

$1442 \%\left(21 \%{ }^{*}\right)$ arylbenzothiophene (Scheme 1, d). ${ }^{9 \mathrm{a}}$ However, the product was obtained in a moderate yield of $47 \%$. Our attempts to extend this synthetic pathway to 4-(benzothiophen-2-yl)benzonitrile was not successful (Scheme 4). The reaction of this 2arylbenzothiophene with 4-bromotoluene or 4bromonitrobenzene afforded the desired coupling products in low yields $(<25 \%)$ according to GC/MS analysis of the crude mixtures. Moreover, the separation of the mono- and di-arylated products was difficult.

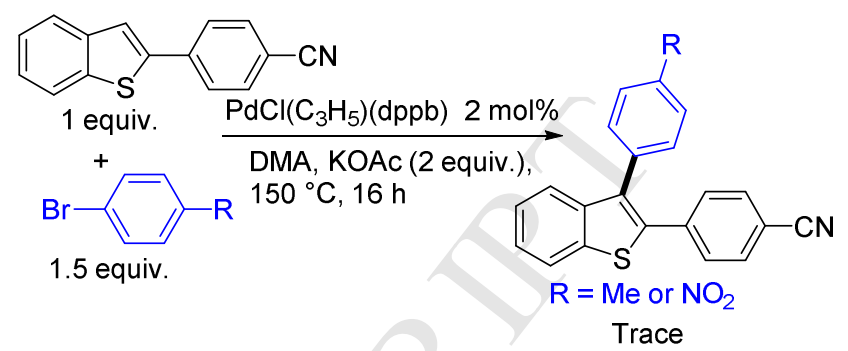

Scheme 4. Attempts of sequential Pd-catalysed direct diarylations of benzothiophene.

Therefore, the access to 2,3-diarylated benzothiophenes via consecutive arylations at $\mathrm{C} 3$ position followed by arylation at C2-position was attempted (Scheme 5).

Scheme 3. Pd-catalysed direct diarylations of benzothiophene.

Consecutive arylations at C2-position followed by arylation at C3-position of benzothiophene could provide an alternative method for the preparation of 2,3-diarylthiophenes. We had previously reported a single example of $\mathrm{C} 3$-arylation of a 2- 


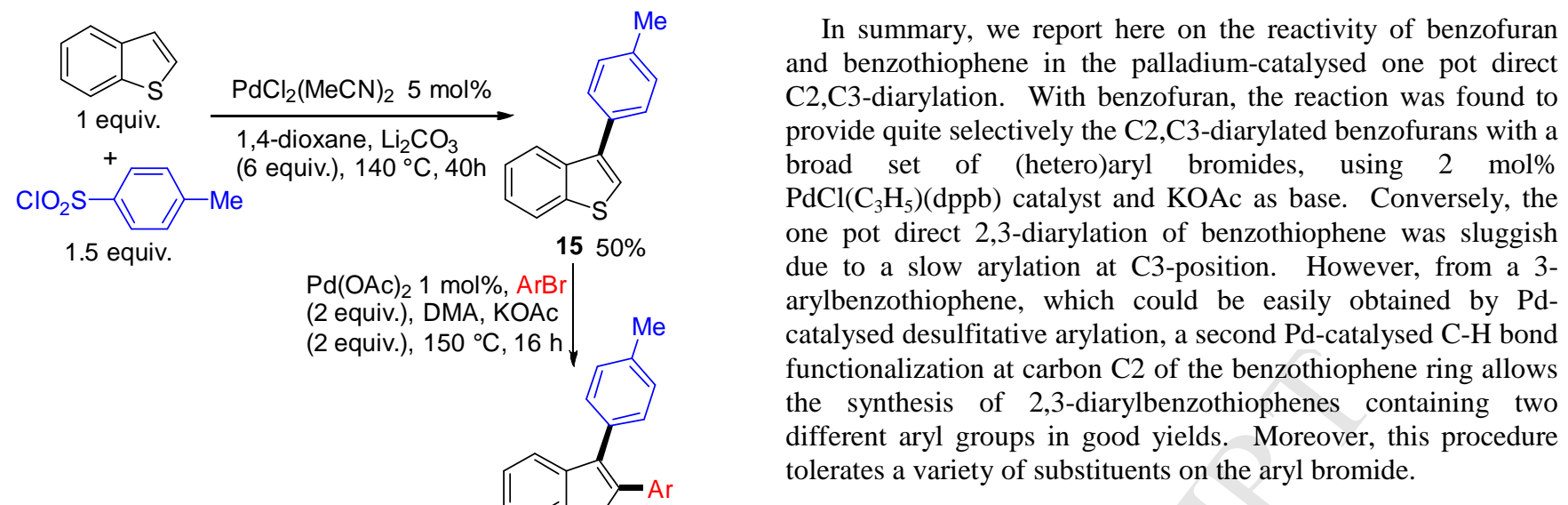

\section{Experimental Section}

\section{General:}

All reactions were carried out under an inert atmosphere with standard Schlenk techniques. ${ }^{1} \mathrm{H}$ NMR spectra were recorded on Bruker GPX $(400 \mathrm{MHz})$ spectrometer. Chemical shifts $(\delta)$ were reported in parts per million relative to residual chloroform (7.26 ppm for ${ }^{1} \mathrm{H}$; $77.0 \mathrm{ppm}$ for ${ }^{13} \mathrm{C}$ ), constants were reported in Hertz. ${ }^{1} \mathrm{H}$ NMR assignment abbreviations were the following: singlet (s), doublet (d), triplet (t), quartet (q), doublet of doublets (dd), doublet of triplets $(\mathrm{dt})$, and multiplet $(\mathrm{m}) .{ }^{13} \mathrm{C}$ NMR spectra were recorded at $100 \mathrm{MHz}$ on the same spectrometer and reported in ppm. All reagents were weighed and handled in air. Compound 15 was prepared according to a reported procedure. ${ }^{12}$

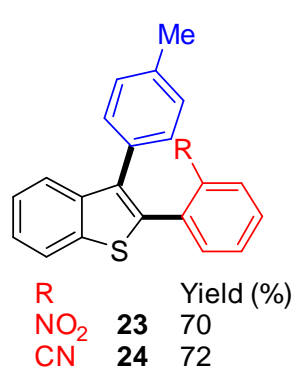

$\begin{array}{lll}\mathrm{CN}^{2} & \mathbf{1 7} & 78\end{array}$

$\mathrm{CHO} 18 \quad 76$

Cl 1972

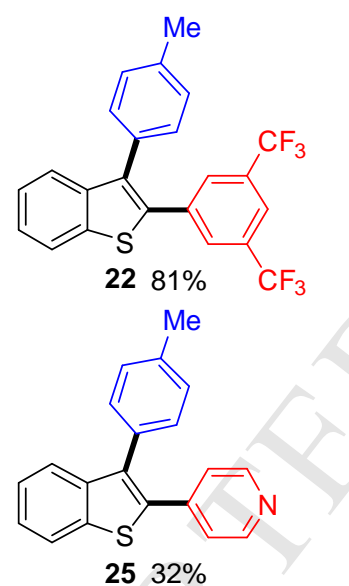

$2532 \%$

Scheme 5. Sequential Pd-catalysed direct diarylations of benzothiophene.

We have recently reported that the Pd-catalysed direct arylation of benzothiophene with benzenesulfonyl chlorides does not proceed via CMD pathway, but probably via the formation of a $\mathrm{Pd}(\mathrm{IV})$ intermediate, affording regioselectively $\mathrm{C} 3$-arylated benzothiophenes. ${ }^{12,13}$ Using $\mathrm{Pd}(\mathrm{MeCN})_{2} \mathrm{Cl}_{2}$ catalyst in the presence of $\mathrm{Li}_{2} \mathrm{CO}_{3}$ in dioxane, and 4-methylbenzenesulfonyl chloride as aryl source, benzothiophene was regioselectively arylated at C3-position to give $\mathbf{1 5}$ in 50\% yield (Scheme 5, top). Then, the C2-arylation of $\mathbf{1 5}$ via CMD process was attempted. Using $1 \mathrm{~mol} \% \mathrm{Pd}(\mathrm{OAc})_{2}$ catalyst with KOAc as the base in DMA during $16 \mathrm{~h}$, and a set of aryl bromides, the desired 2,3-diarylated benzothiophenes 16-25 were obtained in good to high yields. For example, the reaction of $\mathbf{1 5}$ with aryl bromides substituted at para-position by nitro, cyano, formyl or chloro groups gave 1619 in $72-83 \%$ yields. Nitrile and formyl meta- or orthosubstituents on aryl bromides were also tolerated; whereas, the reaction with 4-bromopyridine afforded 25 in a lower yield.

Preparation of the $\operatorname{PdCl}\left(\mathbf{C}_{3} \mathbf{H}_{5}\right)(\mathbf{d p p b})$ catalyst: ${ }^{14}$ An ovendried $40 \mathrm{~mL}$ Schlenk tube equipped with a magnetic stirring bar under argon atmosphere, was charged with $\left[\mathrm{Pd}\left(\mathrm{C}_{3} \mathrm{H}_{5}\right) \mathrm{Cl}\right]_{2}(182$ $\mathrm{mg}, 0.5 \mathrm{mmol})$ and dppb (426 mg, $1 \mathrm{mmol}) .10 \mathrm{~mL}$ of anhydrous dichloromethane were added, then, the solution was stirred at room temperature for twenty minutes. The solvent was removed in vacuum. The yellow powder was used without purification. ${ }^{31} \mathrm{P}$ NMR $\left(81 \mathrm{MHz}, \mathrm{CDCl}_{3}\right) \delta=19.3(\mathrm{~s})$.

General procedure for one pot synthesis of C2,C3-diarylated benzofurans and benzothiophenes 1-14

To a $25 \mathrm{~mL}$ oven dried Schlenk tube, aryl bromide (3 mmol), benzofuran or benzothiophene $(1 \mathrm{mmol})$, KOAc (0.294 g, $3 \mathrm{mmol})$, DMA (2 mL) and $\mathrm{PdCl}\left(\mathrm{C}_{3} \mathrm{H}_{5}\right)(\mathrm{dppb})$ (12.2 $\mathrm{mg}, 0.02 \mathrm{mmol})$ were successively added. The reaction mixture was evacuated by vacuum-argon cycles $(5$ times) and stirred at $150{ }^{\circ} \mathrm{C}$ (oil bath temperature) for 16 hours. After cooling the reaction at room temperature and concentration, the crude mixture was purified by silica column chromatography to afford the diarylated benzofurans or benzothiophenes.

\section{4,4'-(Benzofuran-2,3-diyl)dibenzonitrile $(1)^{8 b}$}

From benzofuran $(0.118 \mathrm{~g}, 1 \mathrm{mmol})$ and 4-bromobenzonitrile $(0.546 \mathrm{~g}, 3 \mathrm{mmol}), 1$ was obtained in $62 \%$ (0.198 g) yield.

${ }^{1} \mathrm{H}$ NMR (400 MHz, $\left.\mathrm{CDCl}_{3}\right): \delta 7.83(\mathrm{~d}, J=8.2 \mathrm{~Hz}, 2 \mathrm{H}), 7.73(\mathrm{~d}$, $J=8.2 \mathrm{~Hz}, 2 \mathrm{H}), 7.69-7.62(\mathrm{~m}, 5 \mathrm{H}), 7.49(\mathrm{~d}, J=8.0 \mathrm{~Hz}, 1 \mathrm{H}), 7.46$ $(\mathrm{t}, J=7.8 \mathrm{~Hz}, 1 \mathrm{H}), 7.34(\mathrm{t}, J=7.8 \mathrm{~Hz}, 1 \mathrm{H})$. 
From benzofuran $(0.118 \mathrm{~g}, 1 \mathrm{mmol})$ and 4-bromobenzaldehyde $(0.555 \mathrm{~g}, 3 \mathrm{mmol}), 2$ was obtained in $63 \%$ (0.205 g) yield.

${ }^{1} \mathrm{H}$ NMR (400 MHz, $\mathrm{CDCl}_{3}$ ): $\delta 10.12(\mathrm{~s}, 1 \mathrm{H}), 10.00(\mathrm{~s}, 1 \mathrm{H}), 8.01$ $(\mathrm{d}, J=8.2 \mathrm{~Hz}, 2 \mathrm{H}), 7.85(\mathrm{~d}, J=8.2 \mathrm{~Hz}, 2 \mathrm{H}), 7.80(\mathrm{~d}, J=8.2 \mathrm{~Hz}$, 2H), $7.70(\mathrm{~d}, J=8.2 \mathrm{~Hz}, 2 \mathrm{H}), 7.61(\mathrm{~d}, J=8.0 \mathrm{~Hz}, 1 \mathrm{H}), 7.52(\mathrm{~d}, J$ $=8.0 \mathrm{~Hz}, 1 \mathrm{H}), 7.43(\mathrm{t}, J=7.8 \mathrm{~Hz}, 1 \mathrm{H}), 7.31(\mathrm{t}, J=7.8 \mathrm{~Hz}, 1 \mathrm{H})$.

${ }^{13} \mathrm{C}$ NMR $\left(100 \mathrm{MHz}, \mathrm{CDCl}_{3}\right): \delta 191.6,191.3,154.4,149.6$, $138.9,135.9,135.7,130.5,130.3,129.9,129.2,128.0,127.4$, $126.0,123.7,120.1,119.0,111.5$.

Elemental analysis: calcd (\%) for $\mathrm{C}_{22} \mathrm{H}_{14} \mathrm{O}_{3}$ (326.35): C 80.97, $\mathrm{H}$ 4.32; found: C 80.69, H 4.48 .

\section{3,3'-(Benzofuran-2,3-diyl)dibenzonitrile (3)}

From benzofuran $(0.118 \mathrm{~g}, 1 \mathrm{mmol})$ and 3-bromobenzonitrile $(0.546 \mathrm{~g}, 3 \mathrm{mmol}), 3$ was obtained in $64 \%$ (0.205 g) yield.

${ }^{1} \mathrm{H}$ NMR (400 MHz, $\mathrm{CDCl}_{3}$ ): $\delta 7.93$ (s, 1H), 7.80-7.69 (m, 4H), 7.67-7.57 (m, 3H), 7.48-7.39 (m, 3H), 7.31 (t, $J=7.8 \mathrm{~Hz}, 1 \mathrm{H})$.

${ }^{13} \mathrm{C}$ NMR $\left(100 \mathrm{MHz}, \mathrm{CDCl}_{3}\right): \delta 154.8,149.2,134.6,134.2$, $133.6,132.6,132.5,131.9,131.3,131.0,130.9,130.2,129.6$, 126.7, 124.4, 120.4, 118.8, 118.7, 117.7, 114.3, 113.9, 112.2.

Elemental analysis: calcd (\%) for $\mathrm{C}_{22} \mathrm{H}_{12} \mathrm{~N}_{2} \mathrm{O}$ (320.35): C 82.49, H 3.78; found: C 82.60, H 3.64.

\section{2,3-Bis(3,5-bis(trifluoromethyl)phenyl)benzofuran (4)}

From benzofuran (0.118 g, $1 \mathrm{mmol})$ and 3,5bis(trifluoromethyl)bromobenzene $(0.879 \mathrm{~g}, 3 \mathrm{mmol}), 4$ was obtained in $83 \%(0.450 \mathrm{~g})$ yield.

${ }^{1} \mathrm{H}$ NMR (400 MHz, $\left.\mathrm{CDCl}_{3}\right): \delta 8.04(\mathrm{~s}, 2 \mathrm{H}), 8.01(\mathrm{~s}, 1 \mathrm{H}), 7.99(\mathrm{~s}$, 2H), $7.84(\mathrm{~s}, 1 \mathrm{H}), 7.66(\mathrm{~d}, J=8.0 \mathrm{~Hz}, 1 \mathrm{H}), 7.50(\mathrm{~d}, J=8.0 \mathrm{~Hz}$, $1 \mathrm{H}), 7.49(\mathrm{t}, J=7.8 \mathrm{~Hz}, 1 \mathrm{H}), 7.37(\mathrm{t}, J=7.8 \mathrm{~Hz}, 1 \mathrm{H})$.

${ }^{13} \mathrm{C}$ NMR $\left(100 \mathrm{MHz}, \mathrm{CDCl}_{3}\right): \delta 154.3,148.1,134.1,133.2(\mathrm{q} J=$ $33.7 \mathrm{~Hz}), 132.5(\mathrm{q} J=33.7 \mathrm{~Hz}), 131.8,129.8,128.6,126.9,126.6$ (m), 124.4, $122.2(\mathrm{q}, J=272.2 \mathrm{~Hz}), 122.1(\mathrm{q}, J=272.2 \mathrm{~Hz})$, 119.9, 117.6, 112.0.

Elemental analysis: calcd (\%) for $\mathrm{C}_{24} \mathrm{H}_{10} \mathrm{~F}_{12} \mathrm{O}$ (542.32): C 53.15, H 1.86; found: C 53.24, H 2.00.

\section{2,2'-(Benzofuran-2,3-diyl)dibenzonitrile (5)}

From benzofuran $(0.118 \mathrm{~g}, 1 \mathrm{mmol})$ and 2-bromobenzonitrile $(0.546 \mathrm{~g}, 3 \mathrm{mmol}), 5$ was obtained in $60 \%$ (0.192 g) yield.

${ }^{1} \mathrm{H}$ NMR $\left(400 \mathrm{MHz}, \mathrm{CDCl}_{3}\right): \delta$ 7.76-7.69 (m, 3H), 7.68-7.64 (m, 2H), 7.62-7.57 (m, 2H), 7.55-7.42 (m, 4H), $7.34(\mathrm{t}, J=7.8 \mathrm{~Hz}$, $1 \mathrm{H})$.

${ }^{13} \mathrm{C}$ NMR $\left(100 \mathrm{MHz}, \mathrm{CDCl}_{3}\right): \delta 154.6,148.9,135.4,134.0$, $133.8,133.2,133.1,132.6,131.5,130.5,129.3,128.4,128.1$, 126.0, 123.6, 120.1, 114.5, 117.4, 117.3, 112.9, 111.7, 111.5.

Elemental analysis: calcd (\%) for $\mathrm{C}_{22} \mathrm{H}_{12} \mathrm{~N}_{2} \mathrm{O}$ (320.35): C 82.49, H 3.78; found: C 82.64, H 3.89.

\section{2,2'-(Benzofuran-2,3-diyl)dibenzaldehyde (6)}

$(0.555 \mathrm{~g}, 3 \mathrm{mmol}), 6$ was obtained in $57 \%$ (0.186 g) yield.

${ }^{1} \mathrm{H}$ NMR $\left(400 \mathrm{MHz}, \mathrm{CDCl}_{3}\right): \delta 10.07(\mathrm{~s}, 1 \mathrm{H}), 9.90(\mathrm{~s}, 1 \mathrm{H}), 7.96$ $(\mathrm{d}, J=8.0 \mathrm{~Hz}, 1 \mathrm{H}), 7.90(\mathrm{~d}, J=8.0 \mathrm{~Hz}, 1 \mathrm{H}), 7.65-7.56(\mathrm{~m}, 2 \mathrm{H})$, 7.53-7.35 (m, 7H), 7.30 (t, $J=7.8 \mathrm{~Hz}, 1 \mathrm{H})$.

${ }^{13} \mathrm{C}$ NMR $\left(100 \mathrm{MHz}, \mathrm{CDCl}_{3}\right): \delta 191.3,191.0,155.2,150.8$, 135.0, 134.9, 134.8, 134.7, 134.1, 132.3, 132.1, 131.4, 130.2, 129.9, 129.3, 129.2, 129.1, 126.4, 124.4, 120.6, 118.0, 112.2.

Elemental analysis: calcd (\%) for $\mathrm{C}_{22} \mathrm{H}_{14} \mathrm{O}_{3}$ (326.35): $\mathrm{C} 80.97, \mathrm{H}$ 4.32; found: C 80.99, H 4.46.

\section{3,3'-(Benzofuran-2,3-diyl)dipyridine $(7)^{7 \mathrm{c}}$}

From benzofuran $(0.118 \mathrm{~g}, 1 \mathrm{mmol})$ and 3-bromopyridine $(0.474$ $\mathrm{g}, 3 \mathrm{mmol}), 7$ was obtained in $68 \%(0.185 \mathrm{~g})$ yield.

${ }^{1} \mathrm{H}$ NMR (400 MHz, $\left.\mathrm{CDCl}_{3}\right): \delta 8.88(\mathrm{bs}, 1 \mathrm{H}), 8.78(\mathrm{bs}, 1 \mathrm{H}), 8.72$ (bs, 1H), 8.57 (bs, 1H), 7.91 (d, J=8.0 Hz, 1H), 7.84 (d, $J=7.7$ $\mathrm{Hz}, 1 \mathrm{H}), 7.61(\mathrm{~d}, J=8.2 \mathrm{~Hz}, 1 \mathrm{H}), 7.49(\mathrm{~d}, J=7.7 \mathrm{~Hz}, 1 \mathrm{H}), 7.48-$ $7.25(\mathrm{~m}, 4 \mathrm{H})$.

\section{6,6'-(Benzofuran-2,3-diyl)bis(2-(trifluoromethyl)pyridine) (8)}

From benzofuran $(0.118 \mathrm{~g}, 1 \mathrm{mmol})$ and 2-bromo-6(trifluoromethyl)pyridine $(0.678 \mathrm{~g}, 3 \mathrm{mmol}), \mathbf{8}$ was obtained in $61 \%(0.249 \mathrm{~g})$ yield.

${ }^{1} \mathrm{H}$ NMR (400 MHz, $\left.\mathrm{CDCl}_{3}\right): \delta 8.19(\mathrm{~d}, J=8.0 \mathrm{~Hz}, 1 \mathrm{H}), 8.16(\mathrm{~d}$, $J=8.0 \mathrm{~Hz}, 1 \mathrm{H}), 7.78-7.90(\mathrm{~m}, 3 \mathrm{H}), 7.69(\mathrm{~d}, J=8.0 \mathrm{~Hz}, 1 \mathrm{H}), 7.59$ $(\mathrm{d}, J=7.8 \mathrm{~Hz}, 2 \mathrm{H}), 7.44(\mathrm{t}, J=7.8 \mathrm{~Hz}, 1 \mathrm{H}), 7.35(\mathrm{t}, J=7.8 \mathrm{~Hz}$, $1 \mathrm{H})$.

${ }^{13} \mathrm{C}$ NMR (100 MHz, $\left.\mathrm{CDCl}_{3}\right): \delta 154.1,152.3,149.5,148.9,147.3$ $(\mathrm{q}, J=34.8 \mathrm{~Hz}), 147.2(\mathrm{q}, J=34.8 \mathrm{~Hz}), 137.9,136.5,129.1$, 128.6, 126.2, 123.7, 123.6, 122.1, 121.0 (q, $J=274.2$ Hz), 120.9 $(\mathrm{q}, J=274.4 \mathrm{~Hz}), 120.3,119.2(\mathrm{~m}), 118.5(\mathrm{~m}), 111.0$.

Elemental analysis: calcd (\%) for $\mathrm{C}_{20} \mathrm{H}_{10} \mathrm{~F}_{6} \mathrm{~N}_{2} \mathrm{O}$ (408.30): $\mathrm{C}$ 58.83, H 2.47; found: C 58.92, H 2.58.

\section{5,5'-(Benzofuran-2,3-diyl)dipyrimidine $(9)^{15}$}

From benzofuran $(0.118 \mathrm{~g}, 1 \mathrm{mmol})$ and 5-bromopyrimidine $(0.477 \mathrm{~g}, 3 \mathrm{mmol}), 9$ was obtained in $73 \%$ (0.200 g) yield.

${ }^{1} \mathrm{H}$ NMR (400 MHz, $\left.\mathrm{CDCl}_{3}\right): \delta 9.34(\mathrm{~s}, 1 \mathrm{H}), 9.20(\mathrm{~s}, 1 \mathrm{H}), 8.97(\mathrm{~s}$, $2 \mathrm{H}), 8.92(\mathrm{~s}, 2 \mathrm{H}), 7.66(\mathrm{~d}, J=8.0 \mathrm{~Hz}, 1 \mathrm{H}), 7.54-7.45(\mathrm{~m}, 2 \mathrm{H})$, $7.37(\mathrm{t}, J=7.8 \mathrm{~Hz}, 1 \mathrm{H})$.

\section{4,4'-(Benzo[b]thiophene-2,3-diyl)dibenzaldehyde (10)}

From benzothiophene $(0.134 \mathrm{~g}, 1 \mathrm{mmol})$ and 4bromobenzaldehyde $(0.555 \mathrm{~g}, 3 \mathrm{mmol}), \mathbf{1 0}$ was obtained in $42 \%$ $(0.144 \mathrm{~g})$ yield.

${ }^{1} \mathrm{H}$ NMR (400 MHz, $\left.\mathrm{CDCl}_{3}\right): \delta 10.07(\mathrm{~s}, 1 \mathrm{H}), 9.98(\mathrm{~s}, 1 \mathrm{H}), 7.97-$ $7.90(\mathrm{~m}, 3 \mathrm{H}), 7.78(\mathrm{~d}, J=8.2 \mathrm{~Hz}, 2 \mathrm{H}), 7.60(\mathrm{~d}, J=8.0 \mathrm{~Hz}, 1 \mathrm{H})$, $7.52(\mathrm{~d}, J=8.2 \mathrm{~Hz}, 2 \mathrm{H}), 7.45(\mathrm{~d}, J=8.2 \mathrm{~Hz}, 2 \mathrm{H}), 7.44-7.38(\mathrm{~m}$, $2 \mathrm{H})$.

${ }^{13} \mathrm{C}$ NMR $\left(100 \mathrm{MHz}, \mathrm{CDCl}_{3}\right): \delta 191.9,191.6,141.7,140.1$, $140.0,139.5,139.2,135.7,135.6,133.7,131.2,130.4,130.3$, $130.0,125.7,125.3,123.5,122.5$. 
Elemental analysis: calcd (\%) for $\mathrm{C}_{22} \mathrm{H}_{14} \mathrm{O}_{2} \mathrm{~S}$ (342.41): C 77.17, H 4.12; found: C 77.14, H 4.21 .

4-(Benzo[b]thiophen-2-yl)benzaldehyde ${ }^{9 \mathrm{a}}$ was also isolated as side-product in $38 \%\left(0.090\right.$ g) yield: ${ }^{1} \mathrm{H}$ NMR $(400 \mathrm{MHz}$, $\left.\mathrm{CDCl}_{3}\right): \delta 9.97(\mathrm{~s}, 1 \mathrm{H}), 7.87(\mathrm{~d}, J=8.3 \mathrm{~Hz}, 2 \mathrm{H}), 7.80(\mathrm{~d}, J=8.3$ $\mathrm{Hz}, 2 \mathrm{H}), 7.80-7.72(\mathrm{~m}, 2 \mathrm{H}), 7.63(\mathrm{~s}, 1 \mathrm{H}), 7.32$ (t, $J=7.3 \mathrm{~Hz}, 1 \mathrm{H})$, $7.28(\mathrm{t}, J=7.3 \mathrm{~Hz}, 1 \mathrm{H})$.

\section{2,3-Bis(3-nitrophenyl)benzo[b]thiophene (11)}

From benzothiophene $(0.134 \mathrm{~g}, 1 \mathrm{mmol})$ and 3bromonitrobenzene $(0.606 \mathrm{~g}, 3 \mathrm{mmol}), 11$ was obtained in $60 \%$ $(0.225 \mathrm{~g})$ yield.

${ }^{1} \mathrm{H}$ NMR (400 MHz, $\left.\mathrm{CDCl}_{3}\right): \delta 8.30(\mathrm{~d}, J=8.0 \mathrm{~Hz}, 1 \mathrm{H}), 8.27(\mathrm{~s}$, $1 \mathrm{H}), 8.21(\mathrm{~s}, 1 \mathrm{H}), 8.17(\mathrm{~d}, J=8.2 \mathrm{~Hz}, 1 \mathrm{H}), 7.97(\mathrm{~d}, J=7.7 \mathrm{~Hz}$, $1 \mathrm{H}), 7.70-7.42(\mathrm{~m}, 7 \mathrm{H})$.

${ }^{13} \mathrm{C}$ NMR $\left(100 \mathrm{MHz}, \mathrm{CDCl}_{3}\right): \delta 148.8,148.5,139.8,139.2$, 138.2 , 136.6, 136.5, 135.5, 135.4, 132.4, 130.2, 129.9, 126.0, $125.5,125.2,124.5,123.2,123.1$ (2C), 122.6.

Elemental analysis: calcd (\%) for $\mathrm{C}_{20} \mathrm{H}_{12} \mathrm{~N}_{2} \mathrm{O}_{4} \mathrm{~S}$ (376.39): $\mathrm{C}$ 63.82, H 3.21; found: C 63.87, H 3.14.

2-(3-Nitrophenyl)benzo[b]thiophene ${ }^{16}$ was also isolated as sideproduct in $12 \%(0.030 \mathrm{~g})$ yield: ${ }^{1} \mathrm{H}$ NMR $\left(400 \mathrm{MHz}, \mathrm{CDCl}_{3}\right): \delta$ $8.55(\mathrm{~s}, 1 \mathrm{H}), 8.18(\mathrm{~d}, J=7.8 \mathrm{~Hz}, 1 \mathrm{H}), 7.99(\mathrm{~d}, J=7.8 \mathrm{~Hz}, 1 \mathrm{H})$, $7.90-7.80(\mathrm{~m}, 2 \mathrm{H}), 7.68(\mathrm{~s}, 1 \mathrm{H}), 7.60(\mathrm{t}, J=8.0 \mathrm{~Hz}, 1 \mathrm{H}), 7.44-$ $7.32(\mathrm{~m}, 2 \mathrm{H})$.

\section{2,2'-(Benzo[b]thiophene-2,3-diyl)dibenzaldehyde (12)}

From benzothiophene $(0.134 \mathrm{~g}, 1 \mathrm{mmol})$ and 2bromobenzaldehyde $(0.555 \mathrm{~g}, 3 \mathrm{mmol}), 12$ was obtained in $31 \%$ $(0.106 \mathrm{~g})$ yield.

${ }^{1} \mathrm{H}$ NMR (400 MHz, $\mathrm{CDCl}_{3}$ ): $\delta 9.94(\mathrm{~s}, 1 \mathrm{H}), 9.75(\mathrm{~s}, 1 \mathrm{H}), 7.93(\mathrm{~d}$, $J=7.7 \mathrm{~Hz}, 1 \mathrm{H}), 7.87(\mathrm{~d}, J=7.0 \mathrm{~Hz}, 1 \mathrm{H}), 7.77(\mathrm{~d}, J=7.5 \mathrm{~Hz}$, $1 \mathrm{H}), 7.60-7.38(\mathrm{~m}, 8 \mathrm{H}), 7.31(\mathrm{~d}, J=7.6 \mathrm{~Hz}, 1 \mathrm{H})$.

${ }^{13} \mathrm{C}$ NMR $\left(100 \mathrm{MHz}, \mathrm{CDCl}_{3}\right): \delta 191.3,190.8,140.6,139.9$, $137.7,136.6,135.2,134.9,134.5,134.0,133.7,132.8,132.5$, $132.2,129.8,129.3,128.8,128.6,126.1,125.9,123.8,122.7$.

Elemental analysis: calcd (\%) for $\mathrm{C}_{22} \mathrm{H}_{14} \mathrm{O}_{2} \mathrm{~S}$ (342.41): C 77.17, H 4.12; found: C 77.21, H 4.02 .

2-(Benzo $[b]$ thiophen-2-yl)benzaldehyde ${ }^{17}$ was also isolated as side-product in $41 \%(0.097 \mathrm{~g})$ yield: ${ }^{1} \mathrm{H}$ NMR $(400 \mathrm{MHz}$, $\left.\mathrm{CDCl}_{3}\right): \delta 10.25(\mathrm{~s}, 1 \mathrm{H}), 8.05(\mathrm{~d}, J=7.7 \mathrm{~Hz}, 1 \mathrm{H}), 7.87(\mathrm{~d}, J=7.0$ $\mathrm{Hz} \mathrm{Hz}, 1 \mathrm{H}), 7.82$ (d, J = 7.0 Hz Hz, 1H), 7.70-7.60 (m, 2H), 7.53 $(\mathrm{d}, J=7.0 \mathrm{~Hz}, \mathrm{~Hz}, 1 \mathrm{H}), 7.45-7.36(\mathrm{~m}, 2 \mathrm{H}), 7.28(\mathrm{~s}, 1 \mathrm{H})$.

\section{3,3'-(Benzo[b]thiophene-2,3-diyl)dipyridine (13)}

From benzothiophene $(0.134 \mathrm{~g}, 1 \mathrm{mmol})$ and 3-bromopyridine $(0.474 \mathrm{~g}, 3 \mathrm{mmol}), \mathbf{1 3}$ was obtained in $38 \%(0.110 \mathrm{~g})$ yield.

${ }^{1} \mathrm{H}$ NMR $\left(400 \mathrm{MHz}, \mathrm{CDCl}_{3}\right): \delta 8.63(\mathrm{dd}, J=4.8,1.5 \mathrm{~Hz}, 1 \mathrm{H})$, $8.58(\mathrm{dd}, J=4.8,1.5 \mathrm{~Hz}, 2 \mathrm{H}), 8.52(\mathrm{dd}, J=4.8,1.5 \mathrm{~Hz}, 1 \mathrm{H})$, $7.92(\mathrm{~d}, J=7.0 \mathrm{~Hz}, 1 \mathrm{H}), 7.67$ (dt, $J=7.8,1.5 \mathrm{~Hz}, 1 \mathrm{H}), 7.61-7.55$ $(\mathrm{m}, 2 \mathrm{H}), 7.47-7.33(\mathrm{~m}, 3 \mathrm{H}), 7.25-7.20(\mathrm{~m}, 1 \mathrm{H})$.

${ }^{13} \mathrm{C}$ NMR $\left(100 \mathrm{MHz}, \mathrm{CDCl}_{3}\right): \delta 151.1,150.3,149.2,149.1$, $140.2,139.4,137.9,137.2,136.9,131.1,131.0,130.0,125.5$, $125.2,123.8,123.5,123.2,122.5$.
Elemental analysis: calcd (\%) for $\mathrm{C}_{18} \mathrm{H}_{12} \mathrm{~N}_{2} \mathrm{~S}$ (288.37): C 74.97, H 4.19; found: C 75.10, H 4.11.

3-(Benzo[b]thiophen-2-yl)pyridine ${ }^{18}$ was also isolated as sideproduct in $36 \%(0.076 \mathrm{~g})$ yield: ${ }^{1} \mathrm{H}$ NMR $\left(400 \mathrm{MHz}, \mathrm{CDCl}_{3}\right): \delta$ $8.99(\mathrm{~s}, 1 \mathrm{H}), 8.57(\mathrm{~d}, J=4.6 \mathrm{~Hz}, 1 \mathrm{H}), 7.96(\mathrm{~d}, J=8.0 \mathrm{~Hz}, 1 \mathrm{H})$, $7.85(\mathrm{~d}, J=7.8 \mathrm{~Hz}, 1 \mathrm{H}), 7.82(\mathrm{~d}, J=7.8 \mathrm{~Hz}, 1 \mathrm{H}), 7.60(\mathrm{~s}, 1 \mathrm{H})$, 7.38-7.34 (m, 3H).

\section{6,6'-(Benzo[b]thiophene-2,3-diyl)bis(2- (trifluoromethyl)pyridine) (14)}

From benzothiophene (0.134 g, $1 \mathrm{mmol})$ and 2-bromo-6(trifluoromethyl)pyridine (0.678 g, $3 \mathrm{mmol}), 14$ was obtained in $42 \%(0.178 \mathrm{~g})$ yield.

${ }^{1} \mathrm{H} \mathrm{NMR}\left(400 \mathrm{MHz}, \mathrm{CDCl}_{3}\right): \delta 7.95(\mathrm{t}, J=7.8 \mathrm{~Hz}, 1 \mathrm{H}), 7.90(\mathrm{~d}, J$ $=8.0 \mathrm{~Hz}, 1 \mathrm{H}), 7.73(\mathrm{~d}, J=8.0 \mathrm{~Hz}, 1 \mathrm{H}), 7.71(\mathrm{~d}, J=8.0 \mathrm{~Hz}, 1 \mathrm{H})$, $7.65(\mathrm{~d}, J=7.9 \mathrm{~Hz}, 1 \mathrm{H}), 7.62(\mathrm{~d}, J=8.0 \mathrm{~Hz}, 1 \mathrm{H}), 7.52(\mathrm{~d}, J=7.7$ $\mathrm{Hz}, 1 \mathrm{H}), 7.46-7.35$ (m, 3H).

${ }^{13} \mathrm{C}$ NMR (100 MHz, $\left.\mathrm{CDCl}_{3}\right): \delta 155.1,152.6,148.3$ (q, $J=34.8$ $\mathrm{Hz}), 147.8(\mathrm{q}, J=34.8 \mathrm{~Hz}), 140.5,139.8,139.6,137.7,137.6$, $133.6,128.3,125.8,125.4,125.0,123.5,122.2,121.2$ (q, $J=$ $274.4 \mathrm{~Hz}), 120.9$ (q, $J=272.8 \mathrm{~Hz}), 119.0,118.8$.

Elemental analysis: calcd (\%) for $\mathrm{C}_{20} \mathrm{H}_{10} \mathrm{~F}_{6} \mathrm{~N}_{2} \mathrm{~S}$ (424.36): C 56.61, $\mathrm{H} 2.38$; found: C 56.80, H 2.30 .

2-(Benzo $[b]$ thiophen-2-yl)-6-(trifluoromethyl)pyridine ${ }^{19}$ was also isolated as side-product in $21 \%(0.058 \mathrm{~g})$ yield: ${ }^{1} \mathrm{H}$ NMR (400 $\left.\mathrm{MHz}, \mathrm{CDCl}_{3}\right): \delta 7.94(\mathrm{~s}, 1 \mathrm{H}), 7.91-7.81(\mathrm{~m}, 4 \mathrm{H}), 7.57(\mathrm{dd}, J=$ 2.2 and $6.4 \mathrm{~Hz}, 1 \mathrm{H}), 7.42-7.36(\mathrm{~m}, 2 \mathrm{H})$.

General procedure for synthesis of C2,C3-diarylated benzothiophenes 16-25

To a $25 \mathrm{~mL}$ oven dried Schlenk tube, aryl bromide (2 mmol), 3(p-tolyl)benzothiophene 15 (0.224 g, $1 \mathrm{mmol}), \mathrm{KOAc}(0.196 \mathrm{~g}, 2$ mmol), DMA (2 mL) and $\mathrm{Pd}(\mathrm{OAc})_{2}(2.24 \mathrm{mg}, 0.01 \mathrm{mmol})$ were successively added. The reaction mixture was evacuated by vacuum-argon cycles (5 times) and stirred at $150{ }^{\circ} \mathrm{C}$ (oil bath temperature) for 16 hours. After cooling the reaction at room temperature and concentration, the crude mixture was purified by silica column chromatography to afford the diarylated benzothiophenes.

\section{2-(4-Nitrophenyl)-3-(p-tolyl)benzo[b]thiophene (16)}

From 3-(p-tolyl)benzothiophene $15(0.224 \mathrm{~g}, 1 \mathrm{mmol})$ and 4bromonitrobenzene $(0.404 \mathrm{~g}, 2 \mathrm{mmol}), 16$ was obtained in $83 \%$ $(0.286 \mathrm{~g})$ yield.

${ }^{1} \mathrm{H}$ NMR (400 MHz, $\left.\mathrm{CDCl}_{3}\right): \delta 8.31(\mathrm{~d}, J=8.5 \mathrm{~Hz}, 2 \mathrm{H}), 7.93(\mathrm{~d}$, $J=8.0 \mathrm{~Hz}, 1 \mathrm{H}), 7.64(\mathrm{~d}, J=8.0 \mathrm{~Hz}, 1 \mathrm{H}), 7.48(\mathrm{~d}, J=8.5 \mathrm{~Hz}$, $2 \mathrm{H}), 7.44(\mathrm{t}, J=7.8 \mathrm{~Hz}, 1 \mathrm{H}), 7.39(\mathrm{t}, J=7.8 \mathrm{~Hz}, 1 \mathrm{H}), 7.24(\mathrm{~d}, J=$ $8.1 \mathrm{~Hz}, 2 \mathrm{H}), 7.22(\mathrm{~d}, J=8.1 \mathrm{~Hz}, 2 \mathrm{H}), 2.45(\mathrm{~s}, 3 \mathrm{H})$.

${ }^{13} \mathrm{C}$ NMR $\left(100 \mathrm{MHz}, \mathrm{CDCl}_{3}\right): \delta 146.9,141.4,140.9,139.4$, $138.1,136.3,136.1,131.8,130.3,130.2,129.9,125.6,125.0$, $124.2,123.8,122.3,21.5$.

Elemental analysis: calcd (\%) for $\mathrm{C}_{21} \mathrm{H}_{15} \mathrm{NO}_{2} \mathrm{~S}$ (345.42): C 73.02, $\mathrm{H}$ 4.38; found: C 73.10, H 4.50. 
From 3-(p-tolyl)benzothiophene $15(0.224 \mathrm{~g}, \mathbf{1} \mathrm{mmol})$ and 4bromobenzonitrile $(0.364 \mathrm{~g}, 2 \mathrm{mmol}), 17$ was obtained in $78 \%$ $(0.253 \mathrm{~g})$ yield.

${ }^{1} \mathrm{H}$ NMR (400 MHz, $\left.\mathrm{CDCl}_{3}\right): \delta 7.88(\mathrm{~d}, J=8.0 \mathrm{~Hz}, 1 \mathrm{H}), 7.61(\mathrm{~d}$, $J=8.0 \mathrm{~Hz}, 1 \mathrm{H}), 7.53(\mathrm{~d}, J=8.5 \mathrm{~Hz}, 2 \mathrm{H}), 7.41(\mathrm{~d}, J=8.5 \mathrm{~Hz}$, 2H), 7.41-7.32 (m, 2H), $7.23(\mathrm{~d}, J=8.1 \mathrm{~Hz}, 2 \mathrm{H}), 7.18(\mathrm{~d}, J=8.1$ $\mathrm{Hz}, 2 \mathrm{H}), 2.43$ (s, 3H).

${ }^{13} \mathrm{C}$ NMR $\left(100 \mathrm{MHz}, \mathrm{CDCl}_{3}\right): \delta 140.9,139.4,139.2,137.9$, $136.7,135.6,132.2,131.8,130.2,130.1,129.9,125.5,124.9$, $124.1,122.3,118.8,111.1,21.5$.

Elemental analysis: calcd (\%) for $\mathrm{C}_{22} \mathrm{H}_{15} \mathrm{NS}$ (325.43): $\mathrm{C} 81.20, \mathrm{H}$ 4.65; found: C 81.41, H 4.47.

\section{4-(3-(p-Tolyl)benzo[b]thiophen-2-yl)benzaldehyde (18)}

From 3-(p-tolyl)benzothiophene $15(0.224 \mathrm{~g}, 1 \mathrm{mmol})$ and 4bromobenzaldehyde $(0.370 \mathrm{~g}, 2 \mathrm{mmol}), \mathbf{1 8}$ was obtained in $76 \%$ $(0.249 \mathrm{~g})$ yield.

${ }^{1} \mathrm{H}$ NMR (400 MHz, $\mathrm{CDCl}_{3}$ ): $\delta 9.97(\mathrm{~s}, 1 \mathrm{H}), 7.89(\mathrm{~d}, J=8.0 \mathrm{~Hz}$, $1 \mathrm{H}), 7.76(\mathrm{~d}, J=8.5 \mathrm{~Hz}, 2 \mathrm{H}), 7.62(\mathrm{~d}, J=8.0 \mathrm{~Hz}, 1 \mathrm{H}), 7.49(\mathrm{~d}, J$ $=8.5 \mathrm{~Hz}, 2 \mathrm{H}), 7.40(\mathrm{t}, J=7.8 \mathrm{~Hz}, 1 \mathrm{H}), 7.35(\mathrm{t}, J=7.8 \mathrm{~Hz}, 1 \mathrm{H})$, 7.24 (d, $J=8.1 \mathrm{~Hz}, 2 \mathrm{H}), 7.21(\mathrm{~d}, J=8.1 \mathrm{~Hz}, 2 \mathrm{H}), 2.42$ (s, 3H).

${ }^{13} \mathrm{C}$ NMR $\left(100 \mathrm{MHz}, \mathrm{CDCl}_{3}\right): \delta 191.8,141.0,140.9,139.3$, $137.8,137.5,135.4,135.2,132.1,130.3,130.2,129.8,129.7$, $125.3,124.8,124.0,122.3,21.5$.

Elemental analysis: calcd (\%) for $\mathrm{C}_{22} \mathrm{H}_{16} \mathrm{OS}$ (328.43): $\mathrm{C} 80.46, \mathrm{H}$ 4.91; found: C 80.47, H 5.04.

\section{2-(4-Chlorophenyl)-3-(p-tolyl)benzo[b]thiophene (19)}

From 3-(p-tolyl)benzothiophene $15(0.224 \mathrm{~g}, 1 \mathrm{mmol})$ and 4bromochlorobenzene $(0.382 \mathrm{~g}, 2 \mathrm{mmol}), 19$ was obtained in $72 \%$ $(0.240 \mathrm{~g})$ yield.

${ }^{1} \mathrm{H}$ NMR (400 MHz, $\left.\mathrm{CDCl}_{3}\right): \delta 7.91(\mathrm{~d}, J=8.0 \mathrm{~Hz}, 1 \mathrm{H}), 7.63(\mathrm{~d}$, $J=8.0 \mathrm{~Hz}, 1 \mathrm{H}), 7.41(\mathrm{t}, J=8.5 \mathrm{~Hz}, 1 \mathrm{H}), 7.38(\mathrm{t}, J=7.8 \mathrm{~Hz}, 1 \mathrm{H})$, 7.32-7.22 (m, 8H), $2.46(\mathrm{~s}, 3 \mathrm{H})$.

${ }^{13} \mathrm{C}$ NMR $\left(100 \mathrm{MHz}, \mathrm{CDCl}_{3}\right): \delta 141.0,138.9,137.9,137.4$, $133.9,133.8,133.1,132.3,130.9,130.3,129.7,128.7,124.9$, 124.7, 123.7, 122.2, 21.5.

Elemental analysis: calcd (\%) for $\mathrm{C}_{21} \mathrm{H}_{15} \mathrm{ClS}$ (334.86): $\mathrm{C} 75.32, \mathrm{H}$ 4.52; found: C 75.17, H 4.34.

\section{2-(3-Nitrophenyl)-3-(p-tolyl)benzo[b]thiophene (20)}

From 3-(p-tolyl)benzothiophene $15(0.224 \mathrm{~g}, 1 \mathrm{mmol})$ and 3bromonitrobenzene $(0.404 \mathrm{~g}, 2 \mathrm{mmol}), \mathbf{2 0}$ was obtained in $80 \%$ $(0.276 \mathrm{~g})$ yield.

${ }^{1} \mathrm{H}$ NMR (400 MHz, $\left.\mathrm{CDCl}_{3}\right): \delta 8.24(\mathrm{t}, J=2.0 \mathrm{~Hz}, 1 \mathrm{H}), 8.11(\mathrm{~d}, J$ $=8.2 \mathrm{~Hz}, 1 \mathrm{H}), 7.92(\mathrm{~d}, J=8.0 \mathrm{~Hz}, 1 \mathrm{H}), 7.64(\mathrm{~d}, J=8.0 \mathrm{~Hz}, 1 \mathrm{H})$, $7.62(\mathrm{~d}, J=8.2 \mathrm{~Hz}, 1 \mathrm{H}), 7.47-7.37(\mathrm{~m}, 3 \mathrm{H}), 7.27(\mathrm{~d}, J=8.1 \mathrm{~Hz}$, 2H), $7.23(\mathrm{~d}, J=8.1 \mathrm{~Hz}, 2 \mathrm{H}), 2.45$ (s, 3H).

${ }^{13} \mathrm{C}$ NMR $\left(100 \mathrm{MHz}, \mathrm{CDCl}_{3}\right): \delta 147.5,140.0,138.2,137.1$, $135.5,135.2,134.6,134.5,130.7,129.3,129.0,128.5,124.5$, 124.0, 123.5, 123.1, 121.5, 121.4, 20.6 .

Elemental analysis: calcd (\%) for $\mathrm{C}_{21} \mathrm{H}_{15} \mathrm{NO}_{2} \mathrm{~S}$ (345.42): C 73.02, H 4.38; found: C 73.00, H 4.21.

\section{3-(3-(p-Tolyl)benzo[b]thiophen-2-yl)benzonitrile (21)}

From 3-(p-tolyl)benzothiophene $15(0.224 \mathrm{~g}, 1 \mathrm{mmol})$ and 3bromobenzonitrile $(0.364 \mathrm{~g}, 2 \mathrm{mmol}), \mathbf{2 1}$ was obtained in $75 \%$ $(0.244 \mathrm{~g})$ yield.

${ }^{1} \mathrm{H}$ NMR (400 MHz, $\mathrm{CDCl}_{3}$ ): $\delta 7.90(\mathrm{~d}, J=8.0 \mathrm{~Hz}, 1 \mathrm{H}), 7.65-$ $7.61(\mathrm{~m}, 2 \mathrm{H}), 7.58-7.51(\mathrm{~m}, 2 \mathrm{H}), 7.47-7.33(\mathrm{~m}, 3 \mathrm{H}), 7.25(\mathrm{~d}, J=$ $8.1 \mathrm{~Hz}, 2 \mathrm{H}), 7.20(\mathrm{~d}, J=8.1 \mathrm{~Hz}, 2 \mathrm{H}), 2.44(\mathrm{~s}, 3 \mathrm{H})$.

${ }^{13} \mathrm{C}$ NMR $\left(100 \mathrm{MHz}, \mathrm{CDCl}_{3}\right): \delta 140.7,138.9,137.8,136.1$, $136.0,135.0,133.8,132.9,131.5,131.0,130.1,129.7,129.2$, $125.2,124.8,123.8,122.2,118.5,112.7,21.4$.

Elemental analysis: calcd (\%) for $\mathrm{C}_{22} \mathrm{H}_{15} \mathrm{NS}$ (325.43): C 81.20, $\mathrm{H}$ 4.65; found: C 81.14, H 4.60.

\section{2-(3,5-Bis(trifluoromethyl)phenyl)-3-(p-} tolyl)benzo[b]thiophene (22)

From 3-(p-tolyl)benzothiophene $15(0.224 \mathrm{~g}, 1 \mathrm{mmol})$ and 3,5bis(trifluoromethyl)bromobenzene (0.586 g, $2 \mathrm{mmol}), 22$ was obtained in $81 \%(0.353 \mathrm{~g})$ yield.

${ }^{1} \mathrm{H}$ NMR (400 MHz, $\left.\mathrm{CDCl}_{3}\right): \delta 7.92(\mathrm{~d}, J=8.0 \mathrm{~Hz}, 1 \mathrm{H}), 7.72(\mathrm{~s}$, $3 \mathrm{H}), 7.65(\mathrm{~d}, J=8.0 \mathrm{~Hz}, 1 \mathrm{H}), 7.44(\mathrm{t}, J=7.8 \mathrm{~Hz}, 1 \mathrm{H}), 7.39(\mathrm{t}, J=$ $7.8 \mathrm{~Hz}, 1 \mathrm{H}), 7.26(\mathrm{~d}, J=8.1 \mathrm{~Hz}, 2 \mathrm{H}), 7.20(\mathrm{~d}, J=8.1 \mathrm{~Hz}, 2 \mathrm{H})$, $2.43(\mathrm{~s}, 3 \mathrm{H})$.

${ }^{13} \mathrm{C}$ NMR (100 MHz, $\left.\mathrm{CDCl}_{3}\right): \delta 141.1,139.4,138.6,137.1$, $136.5,135.6,132.1(\mathrm{q}, J=33.4 \mathrm{~Hz}), 131.7,130.4,130.3,129.8$ (m), 126.0, 125.4, 124.5, 123.5 (q, $J=273.0 \mathrm{~Hz}), 122.1,121.4$ (m), 21.7.

Elemental analysis: calcd (\%) for $\mathrm{C}_{23} \mathrm{H}_{14} \mathrm{~F}_{6} \mathrm{~S}$ (436.42): C 63.30, H 3.23; found: C 63.47, H 3.08.

\section{2-(2-Nitrophenyl)-3-(p-tolyl)benzo[b]thiophene (23)}

From 3-(p-tolyl)benzothiophene 15 (0.224 g, $1 \mathrm{mmol})$ and 2bromonitrobenzene $(0.404 \mathrm{~g}, 2 \mathrm{mmol}), \mathbf{2 3}$ was obtained in $70 \%$ $(0.241 \mathrm{~g})$ yield.

${ }^{1} \mathrm{H}$ NMR (400 MHz, $\left.\mathrm{CDCl}_{3}\right): \delta 7.89(\mathrm{~d}, J=8.0 \mathrm{~Hz}, 1 \mathrm{H}), 7.82(\mathrm{~d}$, $J=8.1 \mathrm{~Hz}, 1 \mathrm{H}), 7.68(\mathrm{~d}, J=8.0 \mathrm{~Hz}, 1 \mathrm{H}), 7.54(\mathrm{t}, J=7.8 \mathrm{~Hz}$, $1 \mathrm{H}), 7.50-7.34(\mathrm{~m}, 4 \mathrm{H}), 7.12(\mathrm{~s}, 4 \mathrm{H}), 2.35(\mathrm{~s}, 3 \mathrm{H})$.

${ }^{13} \mathrm{C}$ NMR $\left(100 \mathrm{MHz}, \mathrm{CDCl}_{3}\right): \delta 150.3,140.1,140.0,137.8$, $136.4,134.3,133.8,132.8,131.4,130.3,129.9,129.7,129.5$, $125.4,125.1,124.9,124.1,122.7,21.7$.

Elemental analysis: calcd (\%) for $\mathrm{C}_{21} \mathrm{H}_{15} \mathrm{NO}_{2} \mathrm{~S}$ (345.42): C 73.02, $\mathrm{H}$ 4.38; found: C 73.12, H 4.47.

\section{2-(3-( $p$-Tolyl)benzo[b]thiophen-2-yl)benzonitrile (24)}

From 3-(p-tolyl)benzothiophene 15 (0.224 g, $1 \mathrm{mmol})$ and 2bromobenzonitrile $(0.364 \mathrm{~g}, 2 \mathrm{mmol}), \mathbf{2 4}$ was obtained in $72 \%$ $(0.234 \mathrm{~g})$ yield.

${ }^{1} \mathrm{H}$ NMR (400 MHz, $\left.\mathrm{CDCl}_{3}\right): \delta 7.90(\mathrm{~d}, J=8.0 \mathrm{~Hz}, 1 \mathrm{H}), 7.75(\mathrm{~d}$, $J=8.2 \mathrm{~Hz}, 1 \mathrm{H}), 7.62(\mathrm{~d}, J=8.0 \mathrm{~Hz}, 1 \mathrm{H}), 7.53(\mathrm{t}, J=7.8 \mathrm{~Hz}$, $1 \mathrm{H}), 7.47(\mathrm{~d}, J=8.0 \mathrm{~Hz}, 1 \mathrm{H}), 7.45-7.36(\mathrm{~m}, 3 \mathrm{H}), 7.18(\mathrm{~d}, J=8.1$ $\mathrm{Hz}, 2 \mathrm{H}), 7.14(\mathrm{~d}, J=8.1 \mathrm{~Hz}, 2 \mathrm{H}), 2.35$ (s, 3H).

${ }^{13} \mathrm{C}$ NMR $\left(100 \mathrm{MHz}, \mathrm{CDCl}_{3}\right): \delta 139.6,139.4,138.2,137.1$, $136.8,133.9,133.2,132.2,132.1,131.0,130.1,129.0,128.2$, $125.0,124.5,123.7,122.1,117.6,113.7,21.1$. 
Elemental analysis: calcd (\%) for $\mathrm{C}_{22} \mathrm{H}_{15} \mathrm{NS}$ (325.43): C 81.20, MANUSBouchu, M. Lemaire, Tetrahedron Lett. 2006, 47, 1015H 4.65; found: C 81.24, H 4.87 .

\section{4-(3-(p-Tolyl)benzo[b]thiophen-2-yl)pyridine (25)}

From 3-(p-tolyl)benzothiophene $15(0.224 \mathrm{~g}, 1 \mathrm{mmol})$ and 4bromopyridine $(0.316 \mathrm{~g}, 2 \mathrm{mmol}), 25$ was obtained in $32 \%$ $(0.096 \mathrm{~g})$ yield.

${ }^{1} \mathrm{H} \mathrm{NMR}\left(400 \mathrm{MHz}, \mathrm{CDCl}_{3}\right): \delta 8.49$ (bs, $\left.2 \mathrm{H}\right), 7.91(\mathrm{~d}, J=8.0 \mathrm{~Hz}$, $1 \mathrm{H}), 7.60(\mathrm{~d}, J=8.0 \mathrm{~Hz}, 1 \mathrm{H}), 7.42(\mathrm{t}, J=7.8 \mathrm{~Hz}, 1 \mathrm{H}), 7.37(\mathrm{t}, J=$ $7.8 \mathrm{~Hz}, 1 \mathrm{H}), 7.26(\mathrm{~d}, J=8.1 \mathrm{~Hz}, 2 \mathrm{H}), 7.25-7.20(\mathrm{~m}, 4 \mathrm{H}), 2.45(\mathrm{~s}$, $3 \mathrm{H})$.

${ }^{13} \mathrm{C}$ NMR $\left(100 \mathrm{MHz}, \mathrm{CDCl}_{3}\right): \delta 149.4,142.4,140.7,138.9$, $137.7,136.0,135.3,131.5,129.8,129.6,125.3,124.6,123.8$, $123.5,122.1,21.2$.

Elemental analysis: calcd (\%) for $\mathrm{C}_{20} \mathrm{H}_{15} \mathrm{NS}$ (301.41): C 79.70, $\mathrm{H}$ 5.02; found: C 79.61, H 4.88.

\section{Acknowledgments}

We thank the Centre National de la Recherche Scientifique and "Rennes Metropole" for providing financial support.

\section{References and notes}

1. A. Ohta, Y. Akita, T. Ohkuwa, M. Chiba, R. Fukunaga, A. Miyafuji, T. Nakata, N. Tani, Y. Aoyagi, Heterocycles 1990, 31, 1951-1958.

2. a) D. Alberico, M. E. Scott, M. Lautens, Chem. Rev. 2007, 107, 174-238; b) T. Satoh, M. Miura, Chem. Lett. 2007, 36, 200-205; c) B.-J. Li, S.-D. Yang, Z.-J. Shi, Synlett 2008, 949-957; d) F. Bellina, R. Rossi, Tetrahedron 2009, 65, 10269-10310; e) L. Ackermann, R. Vicente, A. Kapdi, Angew. Chem. Int. Ed. 2009, 48, 9792-9826; f) X. Chen, K. M. Engle, D.-H. Wang, J.-Q. Yu, Angew. Chem. Int. Ed. 2009, 48, 5094-5115; g) L. Joucla, L. Djakovitch, $A d v$. Synth. Catal. 2009, 351, 673-714; h) C.-L. Sun, B.-J. Li, Z.-J. Shi, Chem. Commun. 2010, 46, 677-685; i) L. Ackermann, Chem. Rev. 2011, 111, 1315-1345; j) N. Kuhl, M. N. Hopkinson, J. Wencel-Delord, F. Glorius, Angew. Chem. Int. Ed. 2012, 51, 10236-10254; k) J. WencelDelord, F. Glorius, Nature Chem. 2013, 5, 369-375; 1) R. Rossi, F. Bellina, M. Lessi, C. Manzini, Adv. Synth. Catal. 2014, 356, 17-117; m) C. B. Bheeter, L. Chen, J.-F. Soulé, H. Doucet, Cat. Sci. Technol. 2016, 6, 2005-2049; n) F. Roudesly, J. Oble, G. Poli, J. Mol. Catal. A: Chem. 2017, 426, 275-296.

3. For selected examples of synthesis of 2-arylbenzofurans via Pd-catalysed direct arylations: a) N. S. Nandurkar, M. J. Bhanushari, M. D. Bhor, B. M. Bhanage, Tetrahedron Lett. 2008, 49, 1045-1048; b) B. Liégaut, D. Lapointe, L. Caron, A. Vlassova, K. Fagnou, J. Org. Chem. 2009, 74, 1826-1834; For C3-arylations of benzofurans: M. Ionita, J. Roger, H. Doucet, ChemSusChem 2010, 3, 367-376.

4. For selected examples of synthesis of 2arylbenzothiophenes via Pd-catalysed direct arylations: a) S. Pivsa-Art, T. Satoh, Y. Kawamura, M. Miura, M. Nomura, Bull. Chem. Soc. Jpn. 1998, 71, 467-473; b) J. Fournier Dit Chabert, L. Joucla, E. David, M. Lemaire, Tetrahedron 2004, 60, 3221-3230; c) M. Nakano, T. Satoh, M. Miura, J. Org. Chem. 2006, 71, 8309-8311; d) J. Fournier Dit Chabert, G. Chatelain, S. Pellet-Rostaing, D.
1018; e) S. Tamba, Y. Okubo, S. Tanaka, D. Monguchi, A. Mori, J. Org. Chem. 2010, 75, 6998-7001; f) D. Lapointe, T. Markiewicz, C. J. Whipp, A. Toderian, K. Fagnou, J. Org. Chem. 2011, 76, 749-759.

5. a) D. García-Cuadrado, A. A. C. Braga, F. Maseras, A. M. Echavarren, J. Am. Chem. Soc., 2006, 128, 1066-1067; b) M. Lafrance, C. N. Rowley, T. K. Woo, K. Fagnou, J. Am. Chem. Soc. 2006, 128, 8754-8756.

6. S. I. Gorelsky, Coord. Chem. Rev. 2013, 257, 153-164.

7. For Pd-catalysed one pot direct C2,C3-diarylations of benzofuran: a) H. A. Chiong, O. Daugulis, Org. Lett. 2007, 9, 1449-1451; b) F. Shibahara, E. Yamaguchi, T. Murai, Chem. Commun. 2010, 46, 2471-2473; c) Y. Li, J. Wang, B. Yan, M. Huang, Y. Zhu, Y. Wu, Y. Wu, Tetrahedron 2015, 71, 2729-2735.

8. For Pd-catalysed sequential direct C2,C3-diarylations of benzofuran: a) L. Loukotova, K. Yuan, H. Doucet, ChemCatChem 2014, 6, 1303-1309; b) T. Dao-Huy, M. Haider, F. Glatz, M. Schnuerch, M. D. Mihovilovic, Eur. J. Org. Chem. 2014, 8119-8125.

9. For Pd-catalysed sequential direct C2,C3-diarylations of benzothiophene: a) L. Zhao, C. Bruneau, H. Doucet, Tetrahedron 2013, 69, 7082-7089; b) F. Abdelmalek, F. Derridj, S. Djebbar, J.-F. Soulé, H. Doucet, Beilstein J. Org. Chem. 2015, 2012-2020: c) Z. Wang, Y. Li, B. Yan, M. Huang, Y. Wu, Synlett 2015, 26, 531-536.

10. For selected examples of synthesis of 2,3diarylbenzofurans via Suzuki coupling: a) T. H. Nguyen, M. Hussain, I. Malik, A. Villinger, P. Langer, Tetrahedron Lett. 2010, 51, 2420-2422; b) C. L. Clouser, J. Chauhan, M. A. Bess, J. L. van Oploo, D. Zhou, S. Dimick-Gray, L. M. Mansky, S. E. Patterson, Bioorg. Med. Chem. Lett. 2012, 22, 6642-6646; c) M. Hussain, N. Thai Hung, N. Abbas, R. A. Khera, I. Malik, T. Patonay, N. Kelzhanova, Z. A. Abilov, A. Villinger, P. Langer, J. Heterocyclic Chem. 2015, 52, 497-505; d) D. D. Vo, M. Elofsson, $A d v$. Synth. Catal. 2016, 358, 4085-4092.

11. For selected examples of synthesis of 2,3diarylbenzothiophenes via Suzuki coupling: a) A. Heynderickx, A. Samat, R. Guglielmetti, Synthesis 2002, 213-216; b) M. Miyasaka, K. Hirano, T. Satoh, M. Miura, Adv. Synth. Catal. 2009, 351, 2683-2688; c) J. Chauhan, A. R. Monteil, S. E. Patterson, Heterocycl. Commun. 2010, 16, 241-244; d) J. H. Jeon, N.-J. Lee, J.-H. Lee, M. C. Suh, Dyes Pigm. 2014, 111, 116-123.

12. K. Yuan, H. Doucet, Chem. Sci., 2014, 5, 392-396.

13. For Pd-catalysed direct arylations or Heck reactions via desulfitative couplings: a) B. Liu, Q. Guo, Y. Cheng, J. Lan, J. You, Chem. Eur. J. 2011, 17, 13415-13419; b) X. Zhou, J. Luo, J. Liu, S. Peng, G.-J. Deng, Org. Lett. 2011, 13, 1432-1435; c) H. Wang, Y. Li, R. Zhang, K. Jin, D. Zhao, C. Duan, J. Org. Chem. 2012, 77, 4849-4853; d) N. Taniguchi, Synlett 2013, 24, 2571-2574; e) S. Liu, Y. Bai, X. Cao, F. Xiao, G.-J. Deng, Chem. Commun. 2013, 49, 7501-7503; f) F. Zhao, Q. Tan, F. Xiao, S. Zhang, G.-J. Deng, Org. Lett. 2013, 15, 1520-1523; g) J. Shi, X.-D. Tang, Y.-C. Wu, H.-N. Li, L.-J. Song, Z.-Y. Wang, Eur. J. Org. Chem. 2015, 1193-1197; h) K. Yuan, J. F. Soulé, H. Doucet, ACS Catal. 2015, 5, 978-991. 
14. T. Cantat, E. Génin, C. Giroud, G. Meyer, A.Jutand, J. MANUSCRIPT Organomet. Chem. 2003, 687, 365-376.

15. A. Arcadi, S. Cacchi, M. D. Rosario, G. Fabrizi, F. Marinelli, J. Org. Chem. 1996, 61, 9280-9288.

16. D. Antonow, T. Marrafa, I. Dawood, T. Ahmed, M. R. Haque, D. E. Thurston, G. Zinzalla, Chem. Commun. 2010, 46, 2289-2291.

17. M. Krzeszewski, D. T. Gryko, J. Org. Chem. 2015, 80, 2893-2899.

18. A. B. Biro, A. Kotschy, Eur. J. Org. Chem. 2007, 13641368.

19. W. Hagui, N. Besbes, E. Srasra, J.-F. Soule, H. Doucet, RSC Adv. 2016, 6, 17110-17117. 


\section{ACCEPTED MANUSCRIPT}

- The reactivity of benzofuran and benzothiophene in C2,C3-diarylation was studied

- A simple one pot access to C2,C3-diarylated benzofuranes is described

- An effective sequential access to C2,C3-diarylated benzothiophene is reported 